\title{
Use of Auto-Injector for Methotrexate Subcutaneous Self-Injections: High Satisfaction Level and Good Compliance in SELF-I Study, a Randomized, Open- Label, Parallel Group Study
}

Alain Saraux · Christophe Hudry · Elena Zinovieva · Hélène Herman-Demars on behalf of Self-I Investigators group

Received: October 30, 2018 / Published online: December 13, 2018

(C) The Author(s) 2018

\section{ABSTRACT}

Introduction: The objective of the study was to compare compliance and acceptability of a new auto-injector (AI) versus syringe for administration of methotrexate (MTX) in patients with rheumatoid arthritis (RA).

Methods: We conducted a randomized, openlabel, parallel group study comparing AI to prefilled syringe (PFS). Adult patients with RA (ACR/EULAR 2010) receiving MTX (orally or by injection) for at least 3 months were allocated to AI or PFS for 6 months and then were allocated to AI for 6 further months. Two co-

The members of Self-I investigators group are listed in acknowledgements.

Enhanced digital features To view enhanced digital features for this article go to https://doi.org/10.6084/ m9.figshare.7388447.

A. Saraux $(\square)$

Rheumatology Department, CHU Brest, Brest,

France

e-mail: alain.saraux@chu-brest.fr

A. Saraux

UMR1227, Lymphocytes B et Autoimmunité,

Inserm, Université de Bretagne occidentale (UBO),

Brest, France

C. Hudry

Rheumatology Institute, Paris, France

E. Zinovieva $\cdot$ H. Herman-Demars

Medical Department, Nordic Pharma SAS, Paris, France primary endpoints were defined at M6: percentage of patients with compliance at least $80 \%$; change in functional capacity assessed by Health Assessment Questionnaire (HAQ). Secondary endpoints included quality of life (RaQoL), RA activity (DAS28), and acceptability. Local safety at injection site was assessed at each visit.

Results: Two-hundred and sixty-five patients were randomized. The main analysis was conducted on per protocol set (99 AI and 98 PFS). Compliance was $96.2 \%$ in AI and $98.9 \%$ in PFS. Good complier rates were $89.9 \%$ and $94.9 \%$, thus a difference of $-5.0 \%(-18.9 \% ; 8.9 \%)$. HAQ remained stable in both groups. No difference was found on RaQoL, change in RA activity, and safety profile. Autonomy, acceptability, and patient satisfaction were better with $\mathrm{AI}$, and patients having had the experience of both AI and PFS preferred AI $(p<0.001)$.

Conclusions: Although this study did not demonstrate non-inferiority of AI versus PFS, compliance was excellent in the two groups, and AI, which was preferred by patients, is a valuable alternative to PFS for administration of MTX.

Trial Registration: ClinicalTrials.gov identifier, NCT02553018.

Funding: Nordic Pharma SAS.

Keywords: Auto-injector;

Methotrexate; Rheumatoid arthritis;

Satisfaction 


\section{INTRODUCTION}

Rheumatoid arthritis (RA) is a chronic inflammatory rheumatic condition involving progressive joint destruction with functional, psychological, and social repercussions, which can be serious for the patient. RA is estimated to affect $0.3-1 \%$ of the general population of adults with approximately 200,000 estimated cases in France [1].

MTX is considered the anchor drug on the basis of its efficacy as a monotherapy and on the basis of its ability to increase the efficacy of biological DMARDs when used in combination $[2,3]$. MTX reduces comorbidities and mortality in RA $[4,5]$. MTX is prescribed in up to $70 \%$ of RA patients [6]. The recommended starting dosage is $10-15 \mathrm{mg} /$ week orally followed by rapid dose escalation (typically 5-mg increment every 1-4 weeks) to achieve the optimal dosage corresponding to $15-25 \mathrm{mg} /$ week in most patients [7].

MTX can also be administered subcutaneously (SC) $[7,8]$. Several studies and systematic literature reviews pointed out better bioavailability, higher efficacy, similar to better tolerability and better compliance of MTX SC as compared to oral MTX [9-12]. Even though this does not mean that all patients should be treated with parenteral MTX, it suggests that patients with an inadequate response and/or intolerance to oral MTX may benefit from parenteral MTX [13].

In recent years, the use of subcutaneous injections gained in importance with the development of prefilled syringes containing MTX. Compared to injection by nursing staff, self-injection can increase patients' treatment adherence and reduce costs for society and patients by decreasing frequency of healthcare professionals' visits and transport. However, reduced manual dexterity, dependence on others, injection site reactions, and injection pain can impair compliance to MTX injections in patients with RA [14]. Manual injections require grip and dexterity to handle the vial, needle, and syringe and its plunger in case. This could be difficult in case of functional limitations due to joint pain and impaired mobility commonly encountered in RA patients. Prefilled auto-injector (AI) technology is a valuable response to such limitations. AIs automatically insert the needle and deliver a controlled and fixed dose of drug. Minimizing the pain at injection site, they are easy to learn and use and RA patients express a high level of satisfaction [15-17]. Few studies have addressed AI effect on RA clinical outcomes [15-17]. They relied mainly on usability and patient preference. Head-to-head comparisons of PFS and AI on RA clinical outcomes are lacking [15].

A newly developed prefilled AI containing MTX has been developed for greater patient convenience and ease of use. This prefilled AI is provided with a needle-cover system to automatically prevent needle stick injury that could potentially result in secondary infections. The aim of this study was to evaluate both compliance and clinical outcomes of this newly developed AI versus prefilled syringes containing MTX in patients suffering from RA.

\section{METHODS}

\section{Compliance with Ethics Guidelines}

This study was conducted in accordance with GCP using the guidance documents and practices offered by the International Conference on Harmonization and the European directives 2001/20/CE and ISO 14155, and in agreement with the local regulations.

All procedures performed in studies involving human participants were in accordance with the Comite de Protection des Personnes (CPP) Ouest VI, an Independent Ethics Committee (IEC) in France where the study was performed, and with the 1964 Helsinki Declaration and its later amendments. Informed consent was obtained from all individual participants included in the study.

The final protocol and its amendments were reviewed and approved by the Competent Authority of France, the French National Agency for Medicines and Health Products Safety (ANSM). A voluntary, signed and dated informed consent form was obtained from the patient prior to any trial-related activity. This 
study was registered under the number NCT02553018 in ClinicalTrials.gov.

\section{Study Design and Patient Population}

This was a multicenter, randomized, comparative, non-inferiority, two-parallel-groups, openlabel clinical study conducted in rheumatology centers in France. The primary objective of the study was to compare the percentage of good compliers and the change in Health Assessment Questionnaire (HAQ) after 6 months of treatment. Secondary objectives encompassed evolution of quality of life after 6 months of treatment, acceptability of the two injection devices, and preference for any of them. The study also aimed at describing local and general safety. The study hypotheses were (1) the compliance with AI would not be inferior to compliance with PFS, (2) effect of MTX administered with AI on HAQ score would not be inferior to those of PFS.

Adult patients with an established diagnosis of RA (according to ACR/EULAR criteria) [15] and receiving MTX (orally of by injection) for at least 3 months entered the study. They were recruited by private practice or hospital-based rheumatologists. For patients currently treated with oral MTX, the switch decided by the rheumatologist was made for either safety, compliance or efficacy issues. Pregnant or breast-feeding women or patients with contraindication to MTX were excluded from the study. Patients were randomly allocated to either MTX by AI or MTX by PFS using a prefilled syringe according to a 1:1 ratio (central randomization).

The investigational product was a disposable, fixed, single-dose auto-injector with $25 \mathrm{~g} / \mathrm{ml}$ MTX. The product was marketed in April of 2017 in France, under the brand name Nordimet $^{\circledR}$ (Nordic Group BV). The reference product was a pre-filled syringe with attached injection needle with $50 \mathrm{mg} / \mathrm{ml}$ MTX (Metoject ${ }^{\circledR}$, medac $\mathrm{GmbH})$. Devices involved similar costs. For instance, in France, prices per milligram of MTX injected (depending on the packaging and out of dispensing fees) range from 1.04 to $2.15 €$ for PFS to 0.88 to $1.86 €$ for AI (http://base-donnees- publique.medicaments.gouv.fr-accessed on 16/11/2018). Patients received one MTX injection per week at a posology varying from 7.5 to $25 \mathrm{mg}$ let at physician's discretion. Different content in mg MTX involved different devices with the corresponding volume to fully inject. Patients received training and instructions for use of the study product by the physician. The allocated treatment was pursued for 6 months, the so-called comparative phase. During a further 6-month non-comparative phase, PFS patients switched to AI. The total study duration per patient was 12 months. At M6 visit, patients who switched from PFS to AI were trained and received instructions for use. During the 6-month comparative phase, patients were asked to visit the physician every other month. During the 6-month non-comparative phase, patients visited the physician at M9 and M12. Products were dispensed by the physician at each visit.

\section{Collected Data}

Collected data included demographics, medical and surgical history, comorbidities, history of RA, and vital signs. Ongoing treatments for RA (DMARDs, corticosteroids, folates) were documented at entry and at M2, M6, and M9 visits.

Compliance was assessed at M2, M6, M9, and M12 visits. It was calculated as the ratio of the actual number of injections divided by the planned number of injections since last visit, based on used and unused products returned by the patient at each visit.

HAQ was collected at entry, M6, and M12 visits. HAQ measures the impact of the disease on the physical function. It includes 20 questions scored using a four-level difficulty scale, organized into eight categories. The HAQ score is the mean of the highest score in each of the eight categories, ranging from 0 to 3 [18].

Quality of life was assessed at entry and at M6 visit by the RAQoL, a 30-item validated disease-specific patient-reported outcome measure [19]. Scores on the RAQoL are a sum of all the individual item scores with a range from 0 to 30 , with a lower score indicating better quality of life. 
RA activity was monitored by the Disease Activity Score (DAS) 28 [20] with ESR (last value available) or CRP if ESR was missing, swollen joints count, tender joints count, morning stiffness, VAS pain, VAS patient's global assessment of disease activity, VAS physician's global assessment of disease activity, extra-articular manifestations. DAS 28 was calculated at entry, M2, M6, M9, and M12 visits.

Patients were asked on acceptability at M6 visit using a self-questionnaire with questions answered with a five-point Likert scale. Acceptability encompassed ease of use, satisfaction, pleasantness, motivation for further utilization, reassurance, treatment-related burden, stress during injection, and pain during injection. Patients were asked to express their preference for AI or PFS at M6 visit for those who had a history of PFS before entry and who were randomized to AI. The same question was asked at M12 for patients who were randomized to PFS during the comparative phase and who switched to AI during the non-comparative phase. Information regarding who performed MTX administration was collected at entry and 6 months later.

Local safety was qualitatively evaluated by the physician at each visit on a four-point verbal scale (unsatisfactory to very satisfactory) and adverse events were collected.

\section{Statistical Analyses}

Results were expressed as mean \pm standard deviation (mean \pm SD) for continuous variables and number and percentage for categorical variables. Percentages were calculated on observed data. Good compliers were defined as patients with at least $80 \%$ compliance over the comparative 6-month period. The response to DMARD was assessed according to the EULAR criteria based on the DAS28 [21]. Percentage of good compliers was estimated with a two-sided 95\% confidence interval (CI) assuming a binomial distribution. Non-inferiority limit was fixed at $-4 \%$ between groups (AI-PFS). For percent change in HAQ from baseline to M6, a one-way analysis of variance model was built using group as fixed effect. Computed means by group were presented as well as an estimate of the difference between means (AI-PFS) and 95\% two-sided confidence interval. Non-inferiority limit was fixed at $-5 \%$ between groups. By convention, percentage change from baseline was set at $400 \%$ for patients with HAQ at baseline $=0$ and HAQ at the end the comparative phase $>0$. This value corresponded to the maximum observed value. Absolute change in HAQ was calculated as (M6-baseline) and compared between groups by a two-way analysis of covariance model with group as fixed effect and baseline score as covariate. Patients overall preference was described at 6 months and 12 months only for patients having used both injection devices. Preference was tested using a Chi-square test for equality of proportions. Other statistical comparisons used Chi-squared tests and Mann-Whitney $U$ tests. As the primary analysis was a non-inferiority analysis, it was conducted in the per protocol (PP) population and confirmed in the full analysis set (FAS) population.

Supposing a 99\% rate of good compliers with PFS and a non-inferiority margin of $4 \%$, with the alpha risk set at 5\%, 130 patients per group were needed (260 in total) to ensure a power of $80 \%$. Supposing a non-inferiority delta of $5 \%$ in percent change in HAQ, 130 patients per group ensured a power of $80 \%$ with alpha risk at $5 \%$ and standard deviation of 0.125 .

The statistical analysis used SAS 9.4 software (SAS Institute, Cary, NC, USA).

\section{RESULTS}

\section{Study Population and Baseline Characteristics}

A total of 271 patients were selected for the study. Six patients attended inclusion visit (V1) but were not randomized due to the non-respect of inclusion/exclusion criteria, resulting in 265 randomized patients by 50 active centers. Eight randomized patients did not receive the study product, resulting in a population of 257 treated patients involved in the safety analysis set. During the comparative phase, 136 patients were allocated to AI and 129 were allocated to 
PFS. Among them, at least one co-primary endpoint was missing in 55 patients, leaving 202 patients in the full analysis set (FAS), 101 in each group. Two patients received PFS instead of AI and three patients received AI instead of PFS. Misallocation was considered as a major protocol violation and these patients were excluded from the PP population which involved 197 patients (99 AI and 98 PFS). Fortyeight patients withdrew from the study during the comparative phase: 26 patients in the AI group and 22 patients in the PFS group. Main reasons for withdrawal during this phase were occurrence of AEs/SAEs ( $n=8$ in AI; $n=5$ in PFS) and patient's request ( $n=6$ in AI; $n=8$ in PFS).

RA has been diagnosed $5.3 \pm 6.6$ years before entry in the study when patients were $58.9 \pm 12.6$ years old. At the inclusion visit, MTX treatment was initiated for $3.1 \pm 3.3$ years; current MTX administration was through subcutaneous route $(58.4 \%)$ or orally $(38.6 \%)$. Mean dosage of MTX was $15.4 \pm 4.2 \mathrm{mg} /$ week corresponding to a mean dose of $0.22 \pm 0.07 \mathrm{mg} / \mathrm{kg} /$ week. Thirty-one patients $(15.5 \%)$ had a current MTX dosage $>0.3 \mathrm{mg} / \mathrm{kg} /$ week. Mean DAS28 score was $3.1 \pm 1.2$. Around one-third of patients were in remission (DAS <2.6). Mean HAQ score was $0.60 \pm 0.60$. Around one-third of patients $(n=56 ; 27.7 \%)$ had no impairment $(\mathrm{HAQ}=0)$. Patients characteristics are detailed in Table 1.

\section{Evolution of the MTX Dose}

At visit M0, patients were prescribed a mean MTX dose of $16.5 \pm 4.2 \mathrm{mg} /$ week thus a mean of $0.24 \pm 0.07 \mathrm{mg} / \mathrm{kg} /$ week, which was inferior to $0.3 \mathrm{mg} / \mathrm{kg} /$ week in $158(79.0 \%)$ patients. Mean dose of MTX remained stable during the comparative phase, ranging from $16.8 \pm 4.2 \mathrm{mg} /$ week at $\mathrm{M} 2$ to $16.9 \pm 4.4 \mathrm{mg} /$ week at M6 (median dose $=15 \mathrm{mg} /$ week). MTX dose changed at least once during the comparative phase in $22(21.8 \%)$ patients with $\mathrm{AI}$ and $16(15.8 \%)$ of those with PFS. Change was mainly due to clinico-biological signs.
Table 1 Patients characteristics

\begin{tabular}{|c|c|c|c|}
\hline $\begin{array}{l}\text { Full analysis } \\
\text { set }\end{array}$ & $\begin{array}{l}\text { Auto- } \\
\text { injector } \\
(N=101)\end{array}$ & $\begin{array}{l}\text { Pre-filled } \\
\text { syringe } \\
(N=101)\end{array}$ & $\begin{array}{l}\text { Overall } \\
(N=202)\end{array}$ \\
\hline Males & $23(22.8 \%)$ & $28(27.7 \%)$ & $51(25.2 \%)$ \\
\hline Age (years) & $58.2 \pm 13.2$ & $59.6 \pm 12.0$ & $58.9 \pm 12.6$ \\
\hline BMI $\left(\mathrm{kg} / \mathrm{m}^{2}\right)$ & $26.3 \pm 5.3$ & $25.9 \pm 4.5$ & $26.1 \pm 4.9$ \\
\hline $\begin{array}{l}\text { Time from RA } \\
\text { diagnosis } \\
\text { (years) }\end{array}$ & $5.2 \pm 6.7$ & $5.3 \pm 6.6$ & $5.3 \pm 6.6$ \\
\hline $\begin{array}{l}\text { Time from } \\
\text { MTX start } \\
\text { (years) }\end{array}$ & $3.3 \pm 3.9$ & $2.9 \pm 2.7$ & $3.1 \pm 3.3$ \\
\hline $\begin{array}{l}\text { MTX route at } \\
\text { entry }\end{array}$ & & & \\
\hline $\begin{array}{l}\text { Subcutaneous } \\
\text { route }\end{array}$ & $55(54.5 \%)$ & $63(62.4 \%)$ & $118(58.4 \%)$ \\
\hline $\begin{array}{l}\text { Intra- } \\
\text { muscular } \\
\text { route }\end{array}$ & $3(3.0 \%)$ & $3(3.0 \%)$ & $6(3.0 \%)$ \\
\hline Oral route & $43(42.6 \%)$ & $35(34.7 \%)$ & $78(38.6 \%)$ \\
\hline $\begin{array}{l}\text { Weekly MTX } \\
\text { dose at entry } \\
(\mathrm{mg})\end{array}$ & $15.0 \pm 4.2$ & $15.8 \pm 4.1$ & $15.4 \pm 4.2$ \\
\hline DAS2 $8^{\mathrm{a}}$ & $3.2 \pm 1.3$ & $3.0 \pm 1.1$ & $3.1 \pm 1.2$ \\
\hline DAS28 $<2.6$ & $33(33.3 \%)$ & $33(34.7 \%)$ & $66(34.0 \%)$ \\
\hline HAQ & $0.67 \pm 0.63$ & $0.54 \pm 0.57$ & $0.60 \pm 0.60$ \\
\hline $\mathrm{RaQoL}^{\mathrm{b}}$ & $8.4 \pm 6.6$ & $7.2 \pm 7.1$ & $7.8 \pm 6.9$ \\
\hline
\end{tabular}

Patients are described according to the allocated treatment. Two patients actually received PFS instead of AI and three patients received AI instead of PFS. Results are expressed as mean \pm SD and $N(\%)$

$B M I$ body mass index, $R A$ rheumatic arthritis, $M T X$ methotrexate

a Missing data for two patients in the AI group and six patients in the PFS group

b Missing data for 22 patients in the AI group and 18 patients in the PFS group 


\section{Compliance}

Compliance during the comparative phase was $96.2 \pm 14.6 \%$ in the AI group and $98.9 \pm 11.4 \%$ in the PFS group, thus a non-significant difference (AI-PFS) of $-2.7 \%(95 \% \mathrm{CI}-6.4 \% ; 1.0 \%)$ in the PP set. Good compliers were $89.9 \%$ in the AI group and $94.9 \%$ in the PFS group, thus a difference of (AI-PFS) of $-5.0 \% \quad(95 \%$ CI $-18.9 \%$; $8.9 \%$ ) which was slightly more than the non-inferiority limit of $-4 \%$. Similar results were observed in FAS set (Table 2).

\section{HAQ Score}

HAQ score remained stable, from baseline to $\mathrm{M} 6$, in the two groups. In the PP set, HAQ score decreased from $0.66 \pm 0.62$ to $0.60 \pm 0.58$ in the AI group and from $0.55 \pm 0.58$ to $0.49 \pm 0.60$ in the PFS group. After imputation to $400 \%$ of relative change in HAQ in patients with baseline value $=0$ and post-baseline value $>0$, percentage of HAQ change at the end of comparative phase from baseline was $21.3 \%$ $(95 \% \mathrm{CI}-2.6 \% ; 45.2 \%)$ in the AI group and $22.4 \%$ (95\% CI $-1.6 \% ; 46.5 \%)$ in the PFS group. Difference of percent change in HAQ score between AI and PFS was - 1.1\% (95\% CI $-35.0 \% ; 32.7 \%)$. The upper limit of the $95 \% \mathrm{CI}$ of the difference was superior to the non-inferiority limit of $5 \%$ but with a high confidence interval due to both missing data for the endpoint and a high variability of the change of HAQ related to imputation rules (Table 3).

Mean absolute change was $-0.06 \pm 0.38$ in the AI group and $-0.06 \pm 0.41$ in the PFS group $(p=0.635)$. Similar results were found in the FAS.

A decrease in HAQ score of -0.22 is considered as the minimal clinically important improvement (MCII) [21]. Twenty-five (25.3\%) patients in the AI group and 30 (30.6\%) in the PFS group improved HAQ score by at least - 0.22. On the other hand, HAQ score increased of at least 0.22 in $19(19.2 \%)$ and 17 (17.3\%) patients, respectively. Using another threshold of -0.375 as MCII [22], 15 (15.2\%) patients in the AI group and 19 (19.4\%) in the PFS group improved HAQ score by at least -0.375 . HAQ score increased of at least 0.375 in $11(11.1 \%)$ and $12(12.2 \%)$ patients, respectively.

\section{RaQoL}

Quality of life, assessed by the RaQoL questionnaire, remained stable in both groups during the comparative phase of the study. No significant difference was found between groups ( $p=0.886$ in the PP set; $p=0.809$ in FAS).

Table 2 Compliance

\begin{tabular}{clllll}
\hline & \multicolumn{2}{l}{ Per protocol $(\boldsymbol{N}=\mathbf{1 9 7})$} & & \multicolumn{2}{l}{ Full analysis set $(\boldsymbol{N}=\mathbf{2 0 2})$} \\
\cline { 2 - 3 } & $\begin{array}{l}\text { Auto-injector } \\
(\boldsymbol{N}=\mathbf{9 9})\end{array}$ & $\begin{array}{l}\text { Pre-filled syringe } \\
(\boldsymbol{N}=\mathbf{9 8})\end{array}$ & & $\begin{array}{l}\text { Auto-injector } \\
(\boldsymbol{N}=\mathbf{1 0 1})\end{array}$ & $\begin{array}{l}\text { Pre-filled syringe } \\
(\boldsymbol{N}=\mathbf{1 0 1})\end{array}$ \\
\hline $\begin{array}{c}\text { Good } \\
\text { compliers }\end{array}$ & $89(89.9 \%)$ & $93(94.9 \%)$ & $91(90.1 \%)$ & $96(95.0 \%)$ \\
& $(82.2 \% ; 95.0 \%)$ & $(88.5 \% ; 98.3 \%)$ & & $(82.5 \% ; 95.1 \%)$ & $(88.8 \% ; 98.4 \%)$ \\
$\begin{array}{c}\text { Compliance } \\
(\%)\end{array}$ & $-5.0 \%(-18.9 \% ; 8.9 \%)$ & & $-5.0 \%(-19.1 \% ; 9.3 \%)$ \\
\hline
\end{tabular}

Good compliers are patients with at least $80 \%$ compliance during the comparative phase. Results are expressed as number (percentage) and two-sided 95\% confidence interval. Contrasts between groups are calculated as (AI-PFS) and are expressed with a two-sided $95 \%$ confidence interval. Non-inferiority threshold was set at $-4 \%$ according to the study protocol 
Table 3 Clinical evolution at the end of the comparative phase (M6)

\begin{tabular}{|c|c|c|c|c|}
\hline & \multicolumn{2}{|l|}{ PP $(N=197)$} & \multicolumn{2}{|l|}{ FAS $(N=202)$} \\
\hline & $\begin{array}{l}\text { Auto-injector } \\
(N=99)\end{array}$ & $\begin{array}{l}\text { Pre-filled syringe } \\
(N=98)\end{array}$ & $\begin{array}{l}\text { Auto-injector } \\
(N=101)\end{array}$ & $\begin{array}{l}\text { Pre-filled syringe } \\
(N=101)\end{array}$ \\
\hline \multicolumn{5}{|l|}{ HAQ score } \\
\hline $\begin{array}{l}\text { Relative change }(\%)-\text { non } \\
\text { imputed values }\end{array}$ & $-7.5 \pm 53.3(0)$ & $-2.2 \pm 80.1(0)$ & $-7.9 \pm 53.0(0)$ & $-3.7 \pm 79.6(0)$ \\
\hline $\begin{array}{l}\text { Relative change } \\
(\%)-\text { imputed values }\end{array}$ & $\begin{array}{l}21.3 \pm 116.9(0) \\
\quad(-2.6 ; 45.2)\end{array}$ & $\begin{array}{l}22.4 \pm 124.2(0) \\
\quad(-1.6 ; 46.5)\end{array}$ & $\begin{array}{l}20.4 \pm 116.0(0) \\
\quad(-3.1 ; 43.9)\end{array}$ & $\begin{array}{l}20.3 \pm 123.1 \\
\quad(-3.2 ; 46.8)\end{array}$ \\
\hline Contrast & \multicolumn{2}{|c|}{$-1.1 \%(-35.0 \% ; 32.7 \%)$} & \multicolumn{2}{|c|}{$0.1 \%(-33.1 \% ; 33.3 \%)$} \\
\hline Absolute change & $-0.06 \pm 0.38(0)$ & $-0.06 \pm 0.41(0)$ & $-0.06 \pm 0.38(0)$ & $-0.06 \pm 0.41(0)$ \\
\hline Contrast & \multicolumn{2}{|l|}{$0.025(-0.08 ; 0.13)$} & \multicolumn{2}{|c|}{$0.031(-0.07 \% ; 0.14 \%)$} \\
\hline \multicolumn{5}{|l|}{ Quality of life } \\
\hline Absolute change in $\mathrm{RaQoL}$ & \multicolumn{2}{|l|}{$p=0.886$} & $p=0.809$ & $0.2 \pm 6.8(0)$ \\
\hline
\end{tabular}

Results are expressed as mean \pm SD (median) (95\% confidence interval). For HAQ, absolute change contrasts were estimated by the difference of least-squares means from a model of analysis of covariance with group as fixed effect and baseline as covariate. For absolute change in $\mathrm{RaQoL}$, comparisons used the Mann-Whitney $U$ test

\section{DAS28}

EULAR good response based in the improvement of the DAS28 score was observed in 25.3 and $23.9 \%$ of patients and moderate response was reported on 20.9 and $22.7 \%$ of patients from of AI group and PFS group respectively, at the end of the comparative phase.

\section{Acceptability}

AI was declared to be not at all or somewhat not stressful by $77.4 \%$ of patients, and not at all or somewhat not painful by $72.6 \%$ as compared with 66.6 and $69.2 \%$ of patients with PFS, respectively. The method of administration was declared to be moderately to very pleasant in 59.4 and $36.8 \%$, reassuring in 91.8 and $88.6 \%$, and easy-to-use in 95.3 and $86.3 \%$ of patients in the AI group and the PFS group respectively. Positive judgments translated in a high rate of moderately to very much satisfied patients (96.5\%), as compared to a rate of $90.1 \%$ of patients in the PFS group. At the end of the comparative phase, patients very or quite motivated to pursue the treatment using the same device represented $83.8 \%$ of the AI group and $69.6 \%$ of the PFS group. Similar results were found for FAS (data not shown).

At the end of the comparative phase, $93.6 \%$ of patients who used a syringe for MTX injection before the study and who were allocated to the AI group preferred auto-injector $(p<0.001)$. At the end of the non-comparative phase, $96.2 \%$ of patients who experienced both methods of injections declared to prefer AI $(p<0.001)$. Overall, products were well accepted but acceptability was better with AI than with PFS. Differences were significant for treatment-related burden $(p=0.018)$, pleasantness $(p<0.001)$, reassurance $(p=0.016)$, and easeof-use $(p<0.001)$. Global satisfaction and willingness to pursue the method of administration was higher for AI than for PFS $(p<0.001$ and $p=0.001)$. No significant difference was observed regarding injection related stress or pain (Fig. 1). 


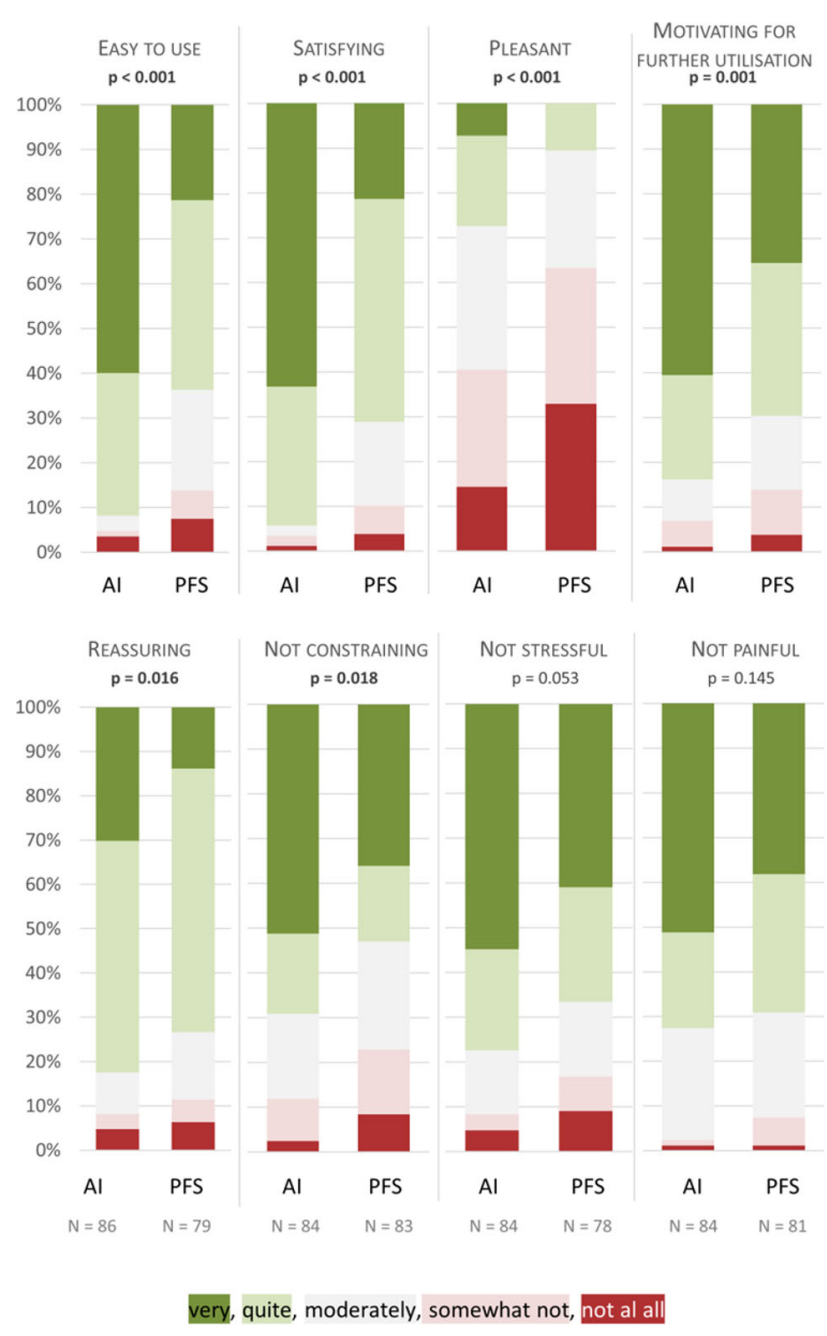

Fig. 1 Acceptability. Patients were asked on acceptability at M6 visit using a self-questionnaire with questions answered with a five-point Likert scale. Acceptability encompassed ease of use, satisfaction, pleasantness,

\section{Patient Autonomy for MTX Administration}

MTX was mainly self-administered: 105 (86.8\%) patients in the AI group and 78 (69.6\%) patients in the PFS group of the safety set, reflecting higher autonomy with AI. Healthcare professionals were involved in MTX administration for eight (6.6\%) patients in the AI group and 22 $(19.6 \%)$ in the PFS group. Family and friends were involved in the remaining cases: i.e., for seven $(5.8 \%)$ and $11(9.8 \%)$ patients, respectively (Fig. 2). motivation for further utilization, reassurance, treatmentrelated burden, stress during injection, and pain during injection. $A I$ auto-injector, $P F S$ prefilled syringe

\section{Safety}

General tolerance was similar between groups. During the comparative phase, nine patients $(6.6 \%)$ from the AI group and four patients (3.3\%) from the PFS group reported local adverse events at injection site, including, for $\mathrm{AI}$, erythema, hematoma, pain, local reaction, and, for PFS, local swelling and skin depigmentation. Difficulty in using the device was reported by two patients in the AI group; a material issue was reported by one patient in the AI group. Local safety was very satisfactory 


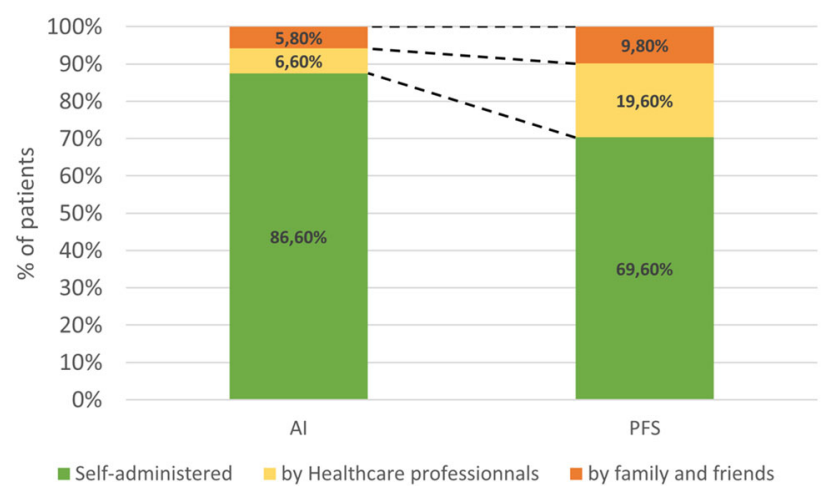

Fig. 2 MTX administration. At M6, patients were asked on whether MTX was self-administered (green) or administered with the help of healthcare professionals (yellow) or family and friends (orange). AI auto-injector, PFS prefilled syringe

or satisfactory according to the physician in 95.5 and $95.8 \%$ at M2, 96.7 and $100 \%$ at M4, and 96.7 and $94.6 \%$ at M6 in the AI group and the PFS group, respectively.

One patient from the PFS group died during the study from a metastatic Merkel's cells carcinoma that was not related to the study product.

\section{DISCUSSION}

This study aimed at assessing the feasibility of MTX self-injections using a pre-filled AI. Based on the percentage of good compliers (overall compliance $\geq 80 \%$ ), the non-inferiority was not demonstrated. Indeed in PP set, the good compliers rate was $89.9 \%$ in the AI group and $94.9 \%$ in the PFS group, and the difference between groups was $-5.0 \%$ (95\% CI - 18.9\%; $8.9 \%$ ) for a non-inferiority limit of $-4 \%$. Nevertheless, compliance during the comparative phase was very good: $96.2 \%$ in the auto-injector group and $98.9 \%$ in the pre-filled syringe injection group. Difference of compliance was $-2.7 \%$ (95\% CI $-6.4 \% ; 1.0 \%)$, a slight difference that did not reach statistical significance. Based on the relative change in HAQ, non-inferiority was not demonstrated. Nevertheless, HAQ score remained stable in the two groups or even slightly improved without difference between groups. A similar proportion of patients experienced a decrease in HAQ at least equal to the minimal clinically important improvement. No difference was found between the two groups on quality of life or evolution of DAS28. Products were well accepted with better patient assessment for AI on treatment-relatedburden, pleasantness, reassurance and ease of use. Global satisfaction and willingness to pursue the treatment were higher for AI.

In patients who had the opportunity to use both methods, 93.6\% declared to prefer autoinjector at M6 and 96.2\% at M12. Self-administration with $\mathrm{AI}$ was more frequent than with PFS.

Proportion of MTX injections involving healthcare professionals in the AI group were about threefold lower than the one in the PFS group, suggesting a higher patient autonomy in the AI group. Safety profile including local safety was similar between the groups.

The World Health Organization considers that improving patients' adherence to longterm therapies is more efficient than any biomedical progress [23]. Non-adherence is not only associated with negative clinical consequences [24] but can also result in substantial healthcare costs. Some authors have highlighted the good usability of SC MTX related to the absence of pain at injection site, a factor contributing to good compliance [25]. According to rheumatologists after a Delphi process, there are three major factors of non-compliance with DMARD: (1) not knowing what to do in the case of an adverse event with DMARD; (2) not having undergone adherence screening by health personnel for early detection of "noncompliant patients"; and (3) not having undergone interventions or strategies that improve 
adherence [26]. Linn et al. [27] documented explicit and implicit attitudes of RA patients towards MTX. If explicit attitudes were positive and health-related, implicit attitudes were, however, negative and sickness-related. Half of the patients displayed explicitly positive but implicitly negative attitudes. Implicit attitudes had a major impact on compliance. Improved adherence has been associated with belief in the necessity and efficacy of MTX, absence of low mood, mildness of disease, and monotherapy with MTX [28]. Compliance to MTX has been reported to be higher with subcutaneous administration when compared to oral route [11]. This could be due to a lower risk of gastrointestinal side effects with parenteral MTX [29].

In the SELF-i study, patients compliance to MTX was greater than $95 \%$. In a recent systematic literature review, Sheiman-Elazari et al. [30] reported a mean compliance rate with DMARDs of $66 \%$ (95\% CI 58\%; 75\%). SELF-I study's better results may be due to the context and to the method of assessment. Patients included in a clinical trial are more prone to be compliant with the study drug; they visit their physician on a regular basis, and the importance of compliance is regularly reminded; in addition, clinical trials are generally brief and do not mimic real-life conditions. Patients were asked to bring back their used and unused treatment units at each visit so that the physician could calculate compliance. Many other definitions of compliance have been found in the literature by Sheiman-Elazari et al. [30]. When using the same definition than in the SELF-i study, compliance rates for DMARDs vary from $68 \%$ [31] to $87 \%$ [32]. Using electronic monitoring systems to record MTX intake (orally or via injection) during 16 weeks, De Cuyper et al. reported that only $58 \%$ of patients were fully adherent [33]. We defined good compliers as patients who had administered at least $80 \%$ of the prescribed MTX units. The same or approaching definitions have been used by others [34, 35]. Curtiss et al. [36] have conducted a systematic review of 12 studies reporting adherence rate to MTX and observed a great variability among patient's characteristics, clinical settings, methods of compliance assessment, and adherence rate. Of the current
MTX users, Aaltonen et al. reported that $6.8 \%$ of patients identified themselves moderately nonadherent to the treatment [37]. As far as we know, compliance to MTX self-injection with a syringe, an AI or any of them has not been specifically evaluated. Consequently, the SELF-I study is one of the first trials addressing the compliance to MTX injection with AI.

The difference between groups in compliance exceeded the non-inferiority threshold that has been set at $-4 \%$. This was a very demanding threshold, largely smaller than usually required. For example, bio-equivalence thresholds are usually (80\%; 125\%). The difference between groups was very small and certainly of no clinical relevance. As an indirect proof, clinical evolution was similar between groups with no difference on HAQ or change in DAS28. Furthermore, mean overall compliance rates in the two groups were both greater than $95 \%$ and were not significantly different [mean difference $-2.9 \%(-6.6 ; 0.7)$; $t$ test $p=0.1097$, in FAS set]. These results suggested that compliance was not impaired when using AI rather than PFS with pre-filled syringes. Only very few patients showed poor compliance $(\leq 60 \%)$ despite general convenience and ease of use of AI and to a lower extent PFS. Device-related aspects may not be the explanation for this slight and likely non-relevant difference in compliance. Moreover, the high level of satisfaction and acceptability and the good local safety did not jeopardize the compliance and certainly did not threaten adherence in a reallife setting. In addition, the large 95\% CI was partly due to a smaller sample size than expected.

The importance of percent change contrasted with the stability of HAQ illustrated by absolute change. Indeed, the variance of the percent change was considerably inflated after imputation of missing data, resulting in a lack of power for comparisons and the large confidence interval of the difference between groups. In this context and for percent change, the study should be considered as non-informative rather than negative since it was not adapted to such variance. HAQ score remained stable during the study. Two definitions of minimal clinically important improvement (MCII) in 
HAQ score have been proposed [22, 38]. Relying on patients self-assessment against other patients, Redelmeier et al. proposed a value of -0.22 [38]. On the other hand, relying on patients' judgment of their current status relative to their previous status, Ward et al. proposed a value of -0.375 [22]. A similar proportion of patients experienced a decrease in HAQ score reaching MCII in both groups, independent of the definition used.

Local safety was found to be very satisfactory or satisfactory by the physician in $96.7 \%$ at M6 in the AI group and in $94.6 \%$ in the PFS group. Given that the administered product was the same in the two groups, differences in local tolerance could have resulted from differences in flow rate, regularity of injection or injected volume. MTX concentration is twice lower in the AI than in the prefilled syringe. Consequently, patients had to inject a twice-larger volume to administer the same MTX dose. Volumes remained however very small. The median dose of MTX was $15 \mathrm{mg} /$ week corresponding to $0.6 \mathrm{ml}$ with the $\mathrm{AI}$ and $0.3 \mathrm{ml}$ with the syringe. Indeed, patients did not report local adverse events that could be related to the injected volume. Using PFS, patients had to push the plunger of the syringe and therefore were able to adapt the flow rate at their own comfort. Conversely, the flow rate was fixed in the auto-injector group. Despite that, injections with AI were not painful. Furthermore, difficulty in using AI was reported only by two patients, although AI was a new device. As PFS was a well-established method of administration, such events were not collected during the study and no comparison could be made.

Fear of needles or at least discomfort with the idea to prick oneself is a widespread feeling. AI have the advantage of hiding the needle and indeed patients from the AI group found the MTX administration less stressful than when using a syringe. In the AI group, the prick was considered to be quite or very stressful by $8.4 \%$ of patients. By contrast, in the PFS group 16.7\% of patients reported that the prick was quite or very stressful. AI was found easy to use and contributed to make the treatment less burdensome. It was considered more pleasant than the syringe. As mentioned earlier, concentration of MTX was different between AI and PFS, resulting in a twofold difference in the volume injected for the same MTX amount. It is not known if this feature had a role in the acceptance of the device. Nonetheless, taking in charge the parameter of injection, AI were found more reassuring. Furthermore, less recourse to nurse support for injection and consequently higher proportion of self-injections illustrated better autonomy, which is a key issue in active patients. Acceptability of AI certainly participated in the improvement of patient autonomy. Furthermore, the reduced involvement of nurse and healthcare staff for AI-related injections may result in potential savings of healthcare resources as both devices per se involved similar costs.

In our study, the majority of patients preferred AI over PFS. However, preference could not be assessed in all patients who went through the study, thus constituting a limitation of the study. Forty-three patients (out of the 202 patients of the FAS population) who entered the study in the AI group, without prior experience with PFS could not have been surveyed. One could not rule out that if they had been switched to PFS, their preference would have been different from the other patients.

\section{CONCLUSIONS}

This study did not demonstrate non-inferiority of AI versus PFS for administration of MTX in RA patients on both compliance and percentage change in HAQ score but compliance was excellent with both devices and HAQ score remained stable in the two groups. In addition, AI was better accepted than PFS, improved autonomy with less healthcare professional resource requirement, and gained patients' preferences. Therefore, AI is a valuable alternative to PFS for MTX self-injections in RA patients.

\section{ACKNOWLEDGEMENTS}

The authors thank all the Self-I investigators for their active participation in the study. The Self-I 
investigators group was composed of (alphabetical order): Dr. Lahouari Aouadi (Lagny Sur Marne), Dr. Azzedine Arif (Argeles Sur Mer), Dr. Heyliette Arty-Hue (Gap), Dr. Frederic Banal (St Mande), Dr. Christopher Banse (Rouen), Dr. Jean-Jacques Baron (Le Havre), Dr. Andre Basch (Caluire Et Cuire), Dr. Valerie Berton (Paris), Dr. Salma Bitar Prud'homme (Paris), Pr Alain Cantagrel (Toulouse), Dr. Pierre Cayla (Lormont), Pr Bernard Combe (Montpellier), Dr. Genevieve Cornaille-Lafage (Paris), Dr. Dominique Duplantier (Orthez), Dr. Martine Elbaz Dellaroli (Avignon), Dr. Veronique Ferrazzi (Montpellier), Pr Rene-Marc Flipo (Lille), Dr. Jean Fulpin (La Crau), Dr. Jean-Claude Gardiol (Sorgues), Dr. Cecile Guilyardi (Nimes), Dr. Anissa Hacene (Castelnaudary), Dr. Christophe Hudry (Paris), Dr. Dominique Jarrige (Millau), Dr. Mireille Jourdan (Lyon), Dr. Herve Laillet (Epinal), Dr. François Lamer (Rennes), Dr. Slim Lassoued (Cahors), Dr. Geraldine Lupo-Mattatia (Nimes), Dr. Eric Marzynski (Erquinghem Lys), Dr. Sylvie Melac-Ducamp (Nevers), Dr. Pierre Monod (Castelnaudary), Dr. Claude Naim (Marseille), Dr. Isabelle Negrier-Chassaing (Limoges), Dr. Pierre Ngasseu (Libourne), Dr. Dominique Plat (Le Mans), Dr. Daniele Prothery (St Paul Les Dax), Dr. Françoise Roch-Bras (Montpellier), Pr Alain Saraux (Brest), Pr Thierry Schaeverbeke (Bordeaux), Dr. Karim Sebaa (Viry-Chatillon), Dr. Eric Senbel (Marseille), Pr Martin Soubrier (Clermont-Ferrand), Dr. Gaëlle SourisseauDiverres (Gujan Mestras), Dr. Dominique Soutif (Tours), Dr. Catherine Straus (Elancourt), Dr. Philippe Tauveron (Tours), Dr. Marie-Agnes Timsit (Brest), Dr. Valerie Vedere (Saint Cyr-SurLoire), Dr. Philippe Viu (Lezignan Corbiere), Dr. Stephanie Werner-Leyval (Rennes). The authors also thank the participants of the study.

Funding. The study and article processing charges were funded by Nordic Pharma SAS. All authors had full access to all of the data in this study and take complete responsibility for the integrity of the data and accuracy of the data analysis.

Medical Writing and Editorial Assistance. The authors thank Pierre Clerson and
Yann Fardini (Soladis Clinical Studies) for assistance in the preparation of the manuscript. This assistance was funded by Nordic Pharma SAS. The authors thank Claire Billard (AdBiostat) for performing the statistical analysis.

Authorship. All named authors meet the International Committee of Medical Journal Editors (ICMJE) criteria for authorship for this article, take responsibility for the integrity of the work as a whole, and have given their approval for this version to be published.

Disclosures. Alain Saraux reports consultancy/speaker fees from Nordic Pharma. Christophe Hudry reports consultancy fees from Nordic Pharma. Elena Zinovieva is a Nordic Pharma employee. Hélène Herman-Demars is a Nordic Pharma employee.

Compliance with Ethics Guidelines. All procedures performed in studies involving human participants were in accordance with the Independent Ethics Committee, Comité de Protection des Personnes (CPP) Ouest VI, and with the 1964 Helsinki Declaration and its later amendments. Informed consent was obtained from all individual participants included in the study.

Data Availability. The datasets generated during and/or analyzed during the current study are not publicly available due to applicable laws and regulations regarding personal data protection and confidentiality.

Open Access. This article is distributed under the terms of the Creative Commons Attribution-NonCommercial 4.0 International License (http://creativecommons.org/licenses/ by-nc/4.0/), which permits any noncommercial use, distribution, and reproduction in any medium, provided you give appropriate credit to the original author(s) and the source, provide a link to the Creative Commons license, and indicate if changes were made. 


\section{REFERENCES}

1. Guillemin F, Saraux A, Guggenbuhl P, et al. Prevalence of rheumatoid arthritis in France: 2001. Ann Rheum Dis. 2005;64(10):1427-30. https://doi.org/ 10.1136/ard.2004.029199.

2. Jacobs JW. Lessons for the use of non-biologic anchor treatments for rheumatoid arthritis in the era of biologic therapies. Rheumatology. 2012;51(suppl_4):iv27-33.

3. Smolen JS, Landewé R, Bijlsma J, et al. EULAR recommendations for the management of rheumatoid arthritis with synthetic and biological diseasemodifying antirheumatic drugs: 2016 update. Ann Rheum Dis. 2017;76:960-77.

4. Choi HK, Hernán MA, Seeger JD, et al. Methotrexate and mortality in patients with rheumatoid arthritis: a prospective study. Lancet. 2002;359(9313):1173-7.

5. Wasko MCM, Dasgupta A, Hubert $\mathrm{H}$, et al. Propensity-adjusted association of methotrexate with overall survival in rheumatoid arthritis. Arthritis Rheumatol. 2013;65(2):334-42.

6. Pincus T, Yazici Y, Sokka T, et al. Methotrexate as the "anchor drug" for the treatment of early rheumatoid arthritis. Clin Exp Rheumatol. 2003;21(5 Suppl 31):S179-85 (published Online First: 2004/02/19).

7. Gaujoux-Viala C, Gossec L, Cantagrel A, et al. Recommendations of the French Society for Rheumatology for managing rheumatoid arthritis. Jt Bone Spine. 2014;81(4):287-97.

8. Smolen JS, Cohen SB, Tony H-P, et al. A randomised, double-blind trial to demonstrate bioequivalence of GP2013 and reference rituximab combined with methotrexate in patients with active rheumatoid arthritis. Ann Rheum Dis. 2017;76(9):1598-602. https://doi.org/10.1136/ annrheumdis-2017-211281.

9. Bianchi G, Caporali R, Todoerti $M$, et al. Methotrexate and rheumatoid arthritis: current evidence regarding subcutaneous versus oral routes of administration. Adv Ther. 2016;33(3):369-78. https://doi.org/10.1007/s12325-016-0295-8 (published Online First: 2016/02/06).

10. Mouterde G, Baillet A, Gaujoux-Viala C, et al. Optimizing methotrexate therapy in rheumatoid arthritis: a systematic literature review. Jt Bone Spine. 2011;78(6):587-92.

11. Cipriani P, Ruscitti P, Carubbi F, et al. Methotrexate in rheumatoid arthritis: optimizing therapy among different formulations. Current and emerging paradigms. Clin Ther. 2014;36(3):427-35. https:// doi.org/10.1016/j.clinthera.2014.01.014 (published Online First: 2014/03/13).

12. Li D, Yang Z, Kang P, et al. Subcutaneous administration of methotrexate at high doses makes a better performance in the treatment of rheumatoid arthritis compared with oral administration of methotrexate: a systematic review and meta-analysis. Semin Arthritis Rheum. 2016;45(6):656-62. https://doi.org/10.1016/j.semarthrit.2015.11.004.

13. Braun J. Optimal administration and dosage of methotrexate. Clin Exp Rheumatol. 2010;28(Suppl 61):S46-51.

14. Whalley D, McKenna SP, de Jong Z, et al. Quality of life in rheumatoid arthritis. Rheumatology. 1997;36(8):884-8. https://doi.org/10.1093/ rheumatology/36.8.884.

15. Demary W, Schwenke H, Rockwitz K, et al. Subcutaneously administered methotrexate for rheumatoid arthritis, by prefilled syringes versus prefilled pens: patient preference and comparison of the selfinjection experience. Patient Preference Adherence. 2014;8:1061-71. https://doi.org/10.2147/PPA. S64111 (published Online First: 2014/08/16).

16. Hudry C, Lebrun A, Moura B, et al. Evaluation of usability and acceptance of a new autoinjector intended for methotrexate subcutaneous self-administration in the management of rheumatoid arthritis. Rheumatol Ther. 2017;4(1):183-94. https://doi.org/10.1007/s40744-017-0057-3 (published Online First: 2017/03/01).

17. Pachon JA, Kivitz AJ, Heuer KU, et al. Assessing usability, label comprehension, pen robustness and pharmacokinetics of a self-administered prefilled autoinjector pen of methotrexate in patients with rheumatoid arthritis. SAGE Open Med. 2014;2: 2050312114564241. https://doi.org/10.1177/20503 12114564241 (published Online First: 2014/01/01).

18. Guillemin F, Braincon S, Pourel J. Measurement of the functional capacity in rheumatoid polyarthritis: a French adaptation of the Health Assessment Questionnaire (HAQ). Revue du rhumatisme et des maladies osteo-articulaires. 1991;58(6):459-65.

19. De Jong Z, Van der Heijde D, McKenna S, et al. The reliability and construct validity of the RAQoL: a rheumatoid arthritis-specific quality of life instrument. Br J Rheumatol. 1997;36(8):878-83.

20. Prevoo M, Van'T Hof MA, Kuper H, et al. Modified disease activity scores that include twenty-eight-joint counts development and validation in a prospective longitudinal study of patients with rheumatoid arthritis. Arthritis Rheumatol. 1995;38(1):44-8. 
21. Ward MM, Guthrie LC, Alba MI. Measures of arthritis activity associated with patient-reported improvement in rheumatoid arthritis when assessed prospectively versus retrospectively. Arthritis Care Res. 2015;67(6):776-81. https://doi.org/10. 1002/acr.22509.

22. Ward MM, Guthrie LC, Alba MI. Clinically important changes in individual and composite measures of rheumatoid arthritis activity: thresholds applicable in clinical trials. Ann Rheum Dis. 2014;74:1691-6.

23. Sabaté E. Adherence to long-term therapies: evidence for action. Geneva: World Health Organization; 2003. https://www.who.int/chp/knowledge/ publications/adherence_report/en/.

24. Pasma A, Schenk CV, Timman R, et al. Non-adherence to disease-modifying antirheumatic drugs is associated with higher disease activity in early arthritis patients in the first year of the disease. Arthritis Res Ther. 2015;17:281. https://doi.org/10. 1186/s13075-015-0801-4 (published Online First: 2015/10/10).

25. Striesow F, Brandt A. Preference, satisfaction and usability of subcutaneously administered methotrexate for rheumatoid arthritis or psoriatic arthritis: results of a postmarketing surveillance study with a high-concentration formulation. Ther Adv Musculoskelet Dis. 2012;4(1):3-9. https://doi. org/10.1177/1759720X11431004 (published Online First: 2012/08/08).

26. Juan Mas A, Castaneda S, Cantero Santamaria JI, et al. Adherence to synthetic disease-modifying antirheumatic drugs in rheumatoid arthritis: results of the OBSERVAR Study. Reumatol Clin. 2017. https://doi.org/10.1016/j.reuma.2017.10.001 (published Online First: 2018/01/01).

27. Linn AJ, Vandeberg L, Wennekers AM, et al. Disentangling rheumatoid arthritis patients' implicit and explicit attitudes toward methotrexate. Front Pharmacol. 2016;7:233. https://doi.org/10. 3389/fphar.2016.00233 (published Online First: 2016/08/26).

28. Hope HF, Bluett J, Barton A, et al. Psychological factors predict adherence to methotrexate in rheumatoid arthritis; findings from a systematic review of rates, predictors and associations with patient-reported and clinical outcomes. RMD Open. 2016;2(1):e000171. https://doi.org/10.1136/ rmdopen-2015-000171 (published Online First: 2016/02/06).

29. Tilling L, Townsend S, David J. Methotrexate and hepatic toxicity in rheumatoid arthritis and psoriatic arthritis. Clin Drug Investig. 2006;26(2):55-62 (published Online First: 2006/12/14).
30. Scheiman-Elazary A, Duan L, Shourt C, et al. The rate of adherence to antiarthritis medications and associated factors among patients with rheumatoid arthritis: a systematic literature review and metaanalysis. J Rheumatol. 2016;43(3):512-23. https:// doi.org/10.3899/jrheum.141371 Online First: 2016/02/18).

(published

31. Harley CR, Frytak JR, Tandon N. Treatment compliance and dosage administration among rheumatoid arthritis patients receiving infliximab, etanercept, or methotrexate. Am J Manag Care. 2003;9(6 Suppl):S136-43 (published Online First: 2003/10/28).

32. Brus HL, van de Laar MA, Taal E, et al. Effects of patient education on compliance with basic treatment regimens and health in recent onset active rheumatoid arthritis. Ann Rheum Dis. 1998;57(3):146-51 (published Online First: 1998/06/26).

33. De Cuyper E, De Gucht V, Maes S, et al. Determinants of methotrexate adherence in rheumatoid arthritis patients. Clin Rheumatol. 2016;35(5):1335-9. https://doi.org/10.1007/ s10067-016-3182-4 (published Online First: 2016/01/20).

34. Pullar T, Peaker S, Martin MF, et al. The use of a pharmacological indicator to investigate compliance in patients with a poor response to antirheumatic therapy. Br J Rheumatol. 1988;27(5):381-4 (published Online First: 1988/10/01).

35. Cannon GW, Mikuls TR, Hayden CL, et al. Merging Veterans Affairs rheumatoid arthritis registry and pharmacy data to assess methotrexate adherence and disease activity in clinical practice. Arthritis Care Res (Hoboken). 2011;63(12):1680-90. https:// doi.org/10.1002/acr.20629 (published Online First: 2011/09/10).

36. Curtis JR, Bykerk VP, Aassi M, et al. Adherence and persistence with methotrexate in rheumatoid arthritis: a systematic review. J Rheumatol. 2016;43(11):1997-2009. https://doi.org/10.3899/ jrheum.151212 (published Online First: 2016/11/03).

37. Aaltonen KJ, Turunen JH, Sokka T, et al. A survey on the medication adherence to methotrexate among rheumatoid arthritis patients treated with self-administered biologic drugs. Clin Exp Rheumatol. 2016;34(4):694-7 (published Online First: 2016/05/24).

38. Redelmeier DA, Lorig K. Assessing the clinical importance of symptomatic improvements: an illustration in rheumatology. Arch Intern Med. 1993;153(11):1337-42. 